\title{
Disseminated mucormycosis due to Cunninghamella bertholletiae in a liver transplant recipient
}

\author{
G.R. Nimmo, R.F. Whiting and R.W. Strong \\ Departments of Clinical Microbiology, Intensive Care and Surgery, Princess Alexandra Hospital, Brisbane, \\ Australia.
}

\begin{abstract}
Summary: Disseminated mucormycosis occurred in a 19 year old female following orthotopic liver transplantation for fulminant Wilson's disease. The causative organism Cunninghamella bertholletiae has previously been described in ten clinical cases, but never before in this setting.
\end{abstract}

\section{Introduction}

Mucormycosis is a recognized complication in patients who are acidotic or who have compromised cellular immune defences due to primary disease or immunosuppressive therapy. It is being increasingly described as an infective complication of organ transplantation. ${ }^{1,2}$ At the same time species only rarely identified as causal agents in the past are being reported with greater frequency. ${ }^{1}$ This report of a case of disseminated infection due to Cunninghamella bertholletiae is the first reported in a liver transplant and illustrates the difficulties encountered in the diagnosis and treatment of this condition.

\section{Case report}

A 19 year old female was admitted deeply jaundiced and encephalopathic. She had a two week history of upper abdominal pain, progressive anaemia and deepening jaundice. A diagnosis of fulminant Wilson's disease was made and it was decided that liver transplantation was the only potentially effective method of treatment. Major clinical problems were acute hepatic failure with encephalopathy, severe coagulopathy, severe intravascular haemolysis and impending renal failure.

She was treated with charcoal haemoperfusion, blood and component therapy and on the fourth day following admission underwent orthotopic liver transplantation. The procedure took 13 hours to perform and blood loss was approximately 51 units

Correspondence: G.R. Nimmo, M.B., B.S., M.Sc., F.R.C.P.A., Department of Clinical Microbiology, Princess Alexandra Hospital, Woolloongabba 4102, Australia

Accepted: 8 July 1987 due mainly to the severe coagulopathy and portal hypertension.

At operation the patient had 3 litres of ascites, a nodular liver weighing 1810 grams and evidence of severe portal hypertension with numerous varices. Histology of the removed liver showed micronodular cirrhosis. The hepatic copper content was $28,200 \mu \mathrm{mol} / \mathrm{kg}$ dry weight (normal range $400-1700$ confirming the diagnosis of Wilson's disease.

Immunosuppressive therapy consisted of cyclo sporin A $4 \mathrm{mg} / \mathrm{kg} /$ day intravenously and methyl prednisolone $50 \mathrm{mg}$ four times daily intravenously, reducing to $20 \mathrm{mg}$ four times daily by 16 th postoperative day. The post-operative course was complicated by oliguric renal failure which responded to fluids, low dose dopamine and frusemide. Bile production by the transplanted liver was poor but liver enzymes and prothrombin time returned to normal limits by the end of the first week.

Chest radiographs initially showed bilateral pulmonary oedema. This had largely cleared by the third day leaving a residual area of consolidation at the right lung base. Diagnoses considered included infection, aspiration and septic embolism. Sputum microscopy revealed Gram negative rods and Gram positive cocci. The leukocyte count was at the upper limit of normal with a relative neutrophilia. The patient was afebrile and was begun on tobramycin $200 \mathrm{mg}$ twice daily and flucloxacillin $1 \mathrm{~g}$ six hourly. Some clearing of the consolidation was noted on the following day's radiograph. Sputum culture grew a heavy growth of Enterobacter sp. and scanty Streptococcus faecalis.

Both hepatic function and the patient's general condition showed a definite improvement by day 6 and an unsuccessful attempt to wean her from

(C) The Fellowship of Postgraduate Medicine, 1988 
mechanical ventilation was made on day 7. She became febrile on the following day and chest radiographs showed worsening of consolidation at the right lung base and new consolidation at the left base. Amoxicillin $1 \mathrm{~g}$ four hourly and metronidazole $500 \mathrm{mg}$ eight hourly were added to her therapy. Aspergillus fumigatus was isolated from a sputum culture taken on day 9 and amphotericin B was commenced as fungal infection was considered probable.

An open lung biopsy was performed on day 11 . The biopsy consisted of a wedge of the left lower lobe as the advent of consolidation in this area had coincided with the onset of fever and general deterioration. Stains and cultures were performed to exclude Pneumocystis carinii, fungi, mycobacteria, Legionella sp. and other bacteria and all proved negative. The histological appearance was consistent with adult respiratory distress syndrome and, although no specific cause was obvious, aspiration was felt to be a likely antecedent.

The patient remained febrile and antifungal therapy was ceased while antibacterial therapy was continued with the substitution of vancomycin for flucloxacillin. After some initial clearing of consolidation on day 12, radiographic appearances continued to worsen as did her general clinical state until the patient died on day 17 post-transplant.

At autopsy fungal infection was found in all lobes of both lungs, with dissemination to the mediastinum, left ventricle, brain, thymus and thyroid. The histological appearance was of broad hyphae with relatively few septae and haphazard branching. Fungal invasion of vessel walls was a noticeable feature. Multiple post-mortem specimens from the lungs and mediastinum grew a grey-white cotton wool-like fungus. Growth was evident at 24 hours on Sabouraud dextrose agar incubated at $25^{\circ} \mathrm{C}$ and $37^{\circ} \mathrm{C}$ and was luxuriant after 72 hours. On subculture, growth also occurred at $42^{\circ} \mathrm{C}$ but was less marked. Hyphae of 7 to $11 \mu \mathrm{m}$ diameter were smooth and hyaline with infrequent septae and branched at acute and right angles. Sporangiophores were vertical with globose to subglobose terminal vesicles bearing spinelike phialides. Conidia were spherical to ellipsoidal and measured 7 to $14 \mu \mathrm{m}$. Based on these criteria the fungus was identified as Cunninghamella bertholletiae, a zygomycete of the order Mucorales.

\section{Discussion}

Mucormycosis is an acute opportunistic mycosis caused by thermotolerant genera of the order
Mucorales of the class Zygomycotina. It is characterized by rapid tissue spread with vascular invasion. The mode of infection would appear to be by inhalation of spores in the case of the rhinocerebral and pulmonary forms, while direct inoculation is thought to account for primary subcutaneous infections. In vitro studies by Waldorf and co-workers ${ }^{3}$ have shown that defective bronchoalveolar macrophage function allows spore germination to occur in diabetic and cortisone treated mice. Dissemination from a primary pulmonary focus is not uncommon.

Infection due to organisms of the genus Cunning hamella is uncommon and only ten cases have been previously reported. ${ }^{5,6}$ Pulmonary involvement was noted in six cases, of which four had disseminated disease. All the patients had an underlying disease likely to compromise immune defences and seven had received steroid therapy. Our case certainly conforms to this pattern. It is, however, the first case reported in a liver transplant recipient and only the second case of mucormycosis in this setting. The previous case would appear to have been diagnosed histologically as no mycological details were recorded.?

In the past there has been some confusion in the literature regarding the classification of clinical isolates of Cunninghamella. In their study of the mating behaviour of Cunninghamella spp., Weitzman and Crist established that Cunninghamella bertholletiae, and not Cunninghamella elegans, is the species causing human infection. ${ }^{8}$ In their subsequent physiological and morphological studies thermotolerance proved to be the key differential feature. ${ }^{9}$ Cunninghamella elegans does not grow at $40^{\circ} \mathrm{C}$.

The difficulty of ante-mortem diagnosis of mucormycosis has been noted previously. ${ }^{10}$ Five of the ten cases of Cunninghamella infection in the literature were diagnosed post-mortem. Biopsy of involved tissue would appear to be the most promising diagnostic approach although it was unsuccessful in this case. The area of the left lobe selected for biopsy had shown the most recent radiological changes, the advent of which had coincided with clinical deterioration. It is disappointing that the tissue biopsied was not representative of the infectious process which must have been active at the time. Realising the obvious difficulties encountered in the timely diagnosis of this condition using current techniques, it would seem that alternative strategies may be needed.

Serological diagnosis of the infections warrants further investigation. Yankey \& Abraham have demonstrated high levels of specific IgA and IgM by immunofluorescence in a patient with cranio- 
facial mucormycosis. ${ }^{11}$ The antigenic profiles of some potentially pathogenic mucoraceous fungi have been compared by Hessian \& Smith $^{12}$ who concluded that the antigenic characterization of the medically significant mucoraceae is feasible. If this proves to be the case then clinically useful tests for specific antibodies and antigens may be a possibility.

The mainstays of therapy for mucormycosis remain surgical resection and systemic amphotericin B. The efficacy of this approach in Cunninghamella infections remains uncertain due to the small

\section{References}

1. Kolbeck, P.C., Makhoul, R.G., Bollinger, R.R. \& Sanfilippo, F. Widely disseminated cunninghamella mucormycosis in an adult renal transplant patient: Case report and review of the literature. Am J Clin Path 1985, 83: 747-753.

2. Dummer, J.S., Hardy, A., Poorsattar, A. \& Ho, M. Early infections in kidney transplant recipients on cyclosporins. Transplant 1983, 36: 259-267.

3. Waldorf, A.R., Levitz, S.M. \& Diamond, R.D. In vivo bronchoalveolar macrophage defense against Rhizopus oryzae and Aspergillus fumigatus. J Infect Dis 1984, 150: $752-760$.

4. Rippon, J.W. Mucormycosis. In: Rippon, J.W. (ed) Medical Mycology: The Pathogenic Fungi and The Pathogenic Actinomycetes. W.B. Saunders, Philadelphia, 1982, pp 615-640.

5. Ventura, G.J., Kantarjian, H.M., Anaissie, E., Hopfer, R.L. \& Fainstein, V. Pneumonia with Cunninghamella species in patients with hematologic malignancies. Cancer 1986, 58: 1534-1536.

6. Sands, J.M., Macher, A.M., Ley, T.J. \& Nienhuis, A.W. Disseminated infection caused by Cunninghamella bertholletiae in a patient with betathalassemia. Ann Intern Med 1985, 102: 59-63. number of cases recorded. Only two patients have survived so far, but it is worth noting that only three of the remaining cases were diagnosed antemortem. Due to the lack of success of the diagnostic efforts in our case, a full course of treatment was not given. However, even if the diagnosis had been made, surgical resection would not have been possible due to the extensive and rapidly progressive nature of the disease. It is doubtful if therapy with amphotericin B alone would have been curative under these circumstances.

7. Wajszczuk, C.P., Dummer, J.S., Ho, M. et al. Fungal infections in liver transplant recipients. Transplant 1985, 40: 347-353.

8. Weitzman, I. \& Crist, M.Y. Studies with clinical isolates of Cunninghamella. I. Mating behaviour. Mycologia 1979, 71: 1024-1033.

9. Weitzman, I. \& Crist, M.Y. Studies with clinical isolates of Cunninghamella. II. Physiological and morphological studies. Mycologia 1980, 72: 661-669.

10. Mackenzie, D.W.R. Rhinocerebral mucormycosis Med J Aust 1983, 1: 300-301.

11. Yankey, R. \& Abraham, A.A. Serological studies of a case of fatal craniofacial mucormycosis. Mycopathologia 1983, 82: 105-109.

12. Hessian, P.A. \& Smith, J.M. Antigenic characterization of some potentially pathogenic mucoraceous fungi. Sabouraudia 1982, 20: 209-216. 